\title{
Comparison of Monomicrobial versus Polymicrobial Candiduria: Time to Awareness of Candiduria
}

\author{
Hyunji Kim, Mi-Kyung Lee, Tae-Hyoung Kim ${ }^{1}$ \\ Departments of Laboratory Medicine and ${ }^{1}$ Urology, Chung-Ang University Hospital, Chung-Ang University College of Medicine, Seoul, Korea
}

Purpose: Candiduria, which is the presence of Candida species in urine, is becoming increasingly common in hospital settings. These normal commensals in humans are often associated with the presence of other microorganisms. In this study, patients presenting with monomicrobial and polymicrobial candiduria were compared.

Materials and Methods: A retrospective study was performed on the demographic, clinical, and laboratory data of 185 patients presenting with candiduria between July 2014 and June 2015 at Chung-Ang University Hospital. The threshold for a positive Candida species urine culture was set to $10^{3} \mathrm{CFU} / \mathrm{ml}$. Data on the following were evaluated: distribution of Candida species; patient age and sex; length of hospital stay; presence of diabetes mellitus (DM), chronic kidney disease (CKD), a urinary catheter, and fever; antibiotic administration; urinalysis; complete blood cells; and C-reactive protein.

Results: Monomicrobial candiduria was more common (128/185, 69.2\%) than polymicrobial candiduria $(57 / 185,30.8 \%)$. The most prevalent species was Candida albicans (monomicrobial vs. polymicrobial candiduria, 61.7\% vs. 54.4\%), followed in order by Candida tropicalis (18.8\% vs. $24.6 \%$ ), and Candida glabrata $(14.8 \%$ vs. $12.3 \%)$, with no significant difference between the two groups. Significant differences in the length of stay, underlying DM or CKD, accompanying symptoms, and urine white blood cells (WBC) and bacterial counts were observed between the two groups $(p<0.05)$.

Conclusions: The length of stay, underlying DM or CKD, accompanying symptoms, and urine WBC and bacterial counts were more associated with polymicrobial candiduria. The early detection and treatment of candiduria will become increasingly important as the Korean population ages.

Keywords: Candida; Urinary tract infections; Candidiasis; Polymicrobial infection

Copyright $\subset$ 2019, Korean Association of Urogenital Tract Infection and Inflammation. All rights reserved. (c) (1) (\&) This is an open access article distributed under the terms of the Creative Commons Attribution Non-Commercial License (http://creativecommons.org/licenses/by-nc/4.0) which permits unrestricted non-commercial use, distribution, and reproduction in any medium, provided the original work is properly cited.
Received: 1 April, 2019

Revised: 25 April, 2019

Accepted: 26 April, 2019

\author{
Correspondence to: Tae-Hyoung Kim \\ (iD) https://orcid.org/0000-0002-0257-3449 \\ Department of Urology, Chung-Ang University \\ College of Medicine, 102 Heukseok-ro, Dongjak- \\ gu, Seoul 06973, Korea \\ Tel: +82-2-6299-1818, Fax: +82-2-6298-8630 \\ E-mail: kthlmk@nate.com
}

\section{INTRODUCTION}

Candida species, which are distributed as normal commensals throughout the human gastrointestinal tract, can cause opportunistic infections in their host. The presence of Candida species in the urine is known as candiduria, the risk factors for which are well known, such as an advanced age, anatomic abnormalities of the urinary tract, concomitant diseases (including uncontrolled diabetes mellitus $[\mathrm{DM}])$, prior use of broad-spectrum antibiotics, the 
presence of a urinary catheter, and female sex [1,2]. The presence of Candida species in the urine can be due to an infection, contamination, or colonization, highlighting the need for a clear distinction. To date, the clinical significance of candiduria and its treatment guidelines have not yet been established [1]. Similarly, the effects of a candiduria treatment on the prognosis of patients are also unknown [3], but the incidence of candiduria is increasing [4,5]. Moreover, reports have shown that candiduria affects patient morbidity and mortality [6-9]. Furthermore, there are reports showing that candiduria can lead to candidemia $[6,10]$. Candiduria is highly undervalued, but the presence of Candida species in the urine can mean extensive candidemia [4]. The rate of progression to invasive candidemia is higher in patients with Candida colonization in the urine than in those without [8].

Candiduria is becoming increasingly common in hospital settings, with a $6.5 \%$ to $25 \%$ increase in prevalence according to the patient's age [4]. The incidence of candiduria in hospitalized patients is increasing, particularly in intensive care units (ICU) [11]. The importance of candiduria is also expected to increase with the gradual increase in average life expectancy and the number of severely ill patients. Therefore, it is imperative to understand the characteristics of patients with candiduria to distinguish true infections that can cause candidemia.

Polymicrobial infections are those caused by a combination of viruses, bacteria, fungi, and other parasites. In these diseases, the microorganisms can exhibit microbial interference interactions (i.e., where one microorganism inhibits the growth of other organisms), or synergistic effects that promote mutual infection [12]. Candiduria is often associated with the presence of other microorganisms. Among patients with candiduria, the rate of candidemia progression is higher in individuals with a polymicrobial infection [8]. Therefore, the analysis of polymicrobial infections among patients with candiduria would help better understand the traits that can lead to candidemia. Therefore, the aim of this study was to compare patients presenting with monomicrobial and polymicrobial candiduria in a tertiary hospital in Korea.

\section{MATERIALS AND METHODS}

\section{Study Design}

This was a retrospective study conducted at Chung-Ang University Hospital. From July 2014 to June 2015, the medical records of patients with Candida species identified in urine cultures were reviewed. The urine culture was carried out as follows. A 1- $\mu$ l of urine was inoculated on blood agar and MacConkey agar plates, which were then incubated for 24 to 48 hours at $37^{\circ} \mathrm{C}$ under an atmosphere containing $5 \% \mathrm{CO}_{2}$. When the culture exceeded $10^{3} \mathrm{CFU} / \mathrm{ml}[13,14]$, the Candida species were isolated by a microscopic examination and Vitek II (bioMérieux Inc., Durham, NC, USA). The patients were divided into two groups according to the number of microorganisms identified in their urine culture, i.e., monomicrobial candiduria and polymicrobial candiduria. For each patient, only the first urine culture results were selected among several urine cultures obtained. Data on the patient's age, length of hospital stay, underlying disease (i.e., DM or chronic kidney disease [CKD]), presence of a urinary catheter, administration of antibiotics in the previous three months, accompanying symptoms (fever, general weakness, voiding difficulty), urinalysis parameters (white blood cells [WBCs] $\geq 10$ /high-power field, bacteria, yeast, Candida, pH), complete blood cell analysis (WBC count, neutrophil-to-lymphocyte ratio), and C-reactive protein level were evaluated along with the urine culture results. The Institutional Review Board of Chung-Ang University Hospital approved this study (IRB no. 1903-008-16255).

\section{Statistical Analysis}

The data are expressed differently according to the characteristics of the variables, where the qualitative variables are expressed as a percentage, and the quantitative variables are expressed as a mean, standard deviation, and range. A Mann-Whitney $U$ test was performed to confirm the significance of the differences between the two groups (i.e., monomicrobial candiduria vs. polymicrobial candiduria). p-values less than 0.05 were considered significant. All statistical analyses were performed using IBM SPSS Statistics for Windows (ver. 22.0; IBM Corp., Armonk, NY, USA). 


\section{RESULTS}

Of the 185 patients included in this study, 128 (69.2\%) had monomicrobial candiduria and 57 (30.8\%) had polymicrobial candiduria. The ratio between male and female in both groups was comparable and the age distribution was similar (Table 1). The distribution of Candida species was similar in the two groups, with the most common species in both groups being Candida albicans, Candida tropicalis, and Candida glabrata (Table 2).

The presence of underlying $\mathrm{DM}$ and $\mathrm{CKD}$ was significantly higher in the polymicrobial candiduria group $(\mathrm{p}=0.025$ and $\mathrm{p}=0.004$, respectively). This group also had longer hospital stays $(\mathrm{p}=0.018$ ), and more accompanying symptoms, such as general weakness and voiding difficulty. On the other hand, there was no significant difference between the groups with regard to the presence of a urinary catheter $(\mathrm{p}=0.425)$. The test results revealed differences in some urinalysis parameters between the two groups. The urinalysis Candida counts were similar in the two groups $(p=0.168)$, but there was a significant difference in the WBC and bacterial counts, with both being higher in those with polymicrobial candiduria ( $p=0.013$ and $p<0.001$, respectively) (Table 1 ).

In the patients with polymicrobial candiduria $(n=57)$, gram-positive bacteria $(n=35,61.4 \%)$ were the most com- mon microorganisms associated with Candida species with Enterococcus faecium ( $\mathrm{n}=23,65.7 \%$ ) being the most common. Six cases of polymicrobial candiduria were encountered, in which two Candida species were identified together. Of these, $C$. albicans (five cases) and $C$. glabrata (four cases) were the most common species identified with other Candida species (Table 3).

\section{DISCUSSION}

The pathophysiology of candiduria can be divided into ascending infections due to microorganisms ascending from the lower urinary tract to the upper urinary tract, and

Table 2. Type and number of Candida species identified in urine cultures

\begin{tabular}{lcc}
\hline Microorganism & $\begin{array}{c}\text { Monomicrobial } \\
\text { candiduria }(\mathrm{n}=128)\end{array}$ & $\begin{array}{c}\text { Polymicrobial } \\
\text { candiduria }(\mathrm{n}=57)\end{array}$ \\
\hline C. albicans & $79(61.7)$ & $31(54.4)$ \\
C. tropicalis & $24(18.8)$ & $14(24.6)$ \\
C. glabrata & $19(14.8)$ & $7(12.3)$ \\
C. parapsilosis & $2(1.6)$ & $1(1.8)$ \\
C. lusitaniae & $2(1.6)$ & $0(0.0)$ \\
C. famata & $1(0.8)$ & $1(1.8)$ \\
C. utilis & $1(0.8)$ & $2(3.5)$ \\
C. krusei & $0(0.0)$ & $1(1.8)$ \\
\hline
\end{tabular}

Values are presented as number of identified (\%).

There were no differences in the distribution of Candida species between the two groups.

Table 1. Clinical characteristics and comparison of the patients with monomicrobial candiduria and polymicrobial candiduria

\begin{tabular}{|c|c|c|c|}
\hline Variable & Monomicrobial candiduria $(n=128)$ & Polymicrobial candiduria $(n=57)$ & p-value $e^{a)}$ \\
\hline Female (\%) & 64.1 & 63.2 & - \\
\hline Age $(y)$ & $71.1 \pm 19.2(2-99)$ & $73.0 \pm 14.2(35-95)$ & 0.718 \\
\hline Length of hospital stay $(\mathrm{d})$ & $62.1 \pm 57.8(3-342)$ & $95.1 \pm 82.1(4-354)$ & 0.018 \\
\hline Diabetes mellitus (\%) & 36.7 & 54.4 & 0.025 \\
\hline Chronic kidney disease (\%) & 7.8 & 21.1 & 0.004 \\
\hline Presence of urinary catheter $(\%)$ & 80.5 & 75.4 & 0.425 \\
\hline Administration of antibiotics (<3 mo) (\%) & 90.6 & 94.7 & 0.345 \\
\hline Fever $(\%)$ & 71.1 & 80.7 & 0.170 \\
\hline General weakness (\%) & 8.6 & 24.6 & 0.003 \\
\hline Voiding difficulty (\%) & 0.0 & 8.8 & 0.001 \\
\hline \multicolumn{4}{|l|}{ Urinalysis } \\
\hline WBCs & $1.3 \pm 1.2(0.0-3.0)$ & $1.7 \pm 1.2(0.0-3.0)$ & 0.013 \\
\hline Bacteria & $1.8 \pm 0.9(0.0-4.0)$ & $2.5 \pm 1.0(0.0-4.0)$ & $<0.001$ \\
\hline Yeast & $2.0 \pm 1.4(0.0-4.0)$ & $2.2 \pm 1.5(0.0-4.0)$ & 0.381 \\
\hline Candida & $1.3 \pm 1.4(0.0-4.0)$ & $1.6 \pm 1.3(0.0-4.0)$ & 0.168 \\
\hline $\mathrm{pH}$ & $5.7 \pm 1.1(5.0-8.5)$ & $5.8 \pm 1.1(5.0-8.0)$ & 0.441 \\
\hline \multicolumn{4}{|l|}{ Complete blood count } \\
\hline WBCs $\left(\times 10^{3} / \mu \mathrm{l}\right)$ & $11.2 \pm 6.1(0.2-47.7)$ & $10.7 \pm 5.8(1.3-34.7)$ & 0.398 \\
\hline Neutrophil-to-lymphocyte ratio (\%) & $10.4 \pm 13.8(0.4-97.0)$ & $8.0 \pm 9.0(0.1-47.7)$ & 0.153 \\
\hline hs-CRP & $80.4 \pm 75.6(0.2-379.8)$ & $62.1 \pm 60.1(0.2-230.3)$ & 0.171 \\
\hline
\end{tabular}

Values are presented as $\%$ only or mean \pm standard deviation (range).

WBCs: white blood cells, hs-CRP: high-sensitivity C-reactive protein.

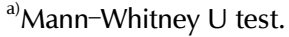


Table 3. Microorganisms associated with polymicrobial candiduria $(n=57)$

\begin{tabular}{lc}
\hline \multicolumn{1}{c}{ Microorganism } & No. of identified (\%) \\
\hline Gram-positive bacteria & \\
Enterococcus faecium & $23(40.4)$ \\
Enterococcus faecalis & $8(14.0)$ \\
Staphylococcus aureus & $3(5.3)$ \\
Enterococcus gallinarum & $1(1.8)$ \\
Gram-negative bacteria & \\
Acinetobacter baumannii & $6(10.5)$ \\
Escherichia coli & $4(7.0)$ \\
Pseudomonas aeruginosa & $3(5.3)$ \\
Acinetobacter lwoffii & $2(3.5)$ \\
Klebsiella pneumoniae & $1(1.8)$ \\
Fungi & \\
Candida albicans-Candida glabrata & $3(5.3)$ \\
C. albicans-Candida tropicalis & $1(1.8)$ \\
C. albicans-Candida kefyr & $1(1.8)$ \\
C. tropicalis-C. glabrata & $1(1.8)$ \\
\hline
\end{tabular}

hematogenous infections caused by the penetration of bloodstream microorganisms into the tissue [15]. Candiduria can be one of the earliest findings of candidemia because it exhibits affinity for the kidneys [16]. A prospective observational study in France reported that $8 \%$ of patients with candiduria developed candidemia, which was caused by the same Candida species [9]. Many studies identified $C$. albicans as the most common species, followed by $C$. glabrata [15]. A comparison of the results of various countries revealed obvious regional differences. After $C$. albicans, C. glabrata was the second most common species in the United States, Spain, and Ethiopia [6,17,18], whereas $C$. tropicalis was the second most prevalent in Korea, Brazil, India, and Turkey $[2,7,19,20]$. In the present study, $C$. albicans, $C$. tropicalis, and $C$. glabrata were identified in this order, which can be explained by geographic differences.

The risk factors for candiduria are well known, and no difference in risk factors between the two groups in this study was found except for the presence of DM and CKD. To the best of the authors' knowledge, there are no reports on the association between monomicrobial candiduria and polymicrobial candiduria. Consequently, previous studies could not be used to explain the difference regarding underlying diseases in these groups. Nevertheless, this analysis is meaningful in terms of disease characteristics. In patients with DM, glycosuria can become a predisposing factor for infections, and the urinary stasis caused by a neurogenic bladder may promote infections and colonization. In addition, a weakened host defense due to im- paired phagocytic activity in patients with DM is believed to be the cause of infection by a range of various microorganisms [15]. Candiduria is often associated with critically ill patients, where a Candida infection influences the renal function, suggesting that a more advanced polymicrobial infection is associated with CKD aggravation.

Polymicrobial candiduria can be accompanied by not only bacterial, but also yeast infections, occurring in approximately $5 \%$ to $10 \%$ of candiduria cases [4,9]. Simpson et al. [3] reported that $28 \%$ of patients hospitalized with asymptomatic candiduria had concomitant bacteriuria that did not affect the morbidity or mortality. On the other hand, Magill et al. [8], who examined the risk factors associated with the development of invasive candidemia according to the location of the Candida colonies in patients in the surgical ICU, reported different results. Invasive candidemia occurred in $5 \%$ of patients, where the colonization of urine by Candida species was confirmed before the diagnosis. Colonization was reported in the following order of prevalence: C. albicans (89\%), C. glabrata (67\%), C. tropicalis (33\%), and Candida parapsilosis (11\%); 89\% of these patients had colonization of two or more Candida species. In another study, cell surface proteins of $E$. faecium were found to be attached to uroepithelial cells, where they have been reported to play a role in urinary tract infections [21]. Enterococcus faecalis, which is known to occur symbiotically with $C$. albicans, inhibited the hyphal morphogenesis of $C$. albicans, thereby affecting its virulence [22]. These results suggest that $E$. faecium is associated most frequently with polymicrobial candiduria because of its affinity to the genitourinary tract and its presence as resident flora. Because $E$. faecalis has a symbiotic relationship with Candida, it is believed to be the second most common cause of polymicrobial candiduria, and it is necessary to confirm the virulence factor to gauge the possible development of candidemia in these patients. In urine cultures with two or more Candida species identified, the combination of $C$. albicans and $C$. glabrata was most common, which was consistent with previous studies. On the other hand, the incidence of non-albicans Candida species has increased recently [19] and resistance to antifungal agents is being reported more frequently [23]. In a multicenter study conducted in South Korea, C. glabrata isolated from the bloodstream was highly resistant to antifungal agents [23]. Therefore, the Candida species identified in the culture 
can help determine if treatment is necessary, and if so, what the treatment options should be. For this reason, care should be taken when identifying $C$. glabrata in the urine.

The limitation of this study was that the number of patients analyzed was small. A larger patient cohort will result in more relevant factors being obtained. Analyses of $C$. albicans and non-albicans Candida species were not conducted because of the small number of patients. Further analysis of non-albicans Candida species that are resistant to treatment and are becoming increasingly prevalent will be needed.

\section{CONCLUSIONS}

These results suggest that the length of hospital stay, presence of DM or CKD, accompanying symptoms, and urine WBC and bacterial counts are more associated with polymicrobial candiduria. Moreover, the early detection and treatment of candiduria will become increasingly important as the Korean population ages. On the other hand, the clinical significance of candiduria and guidelines for its treatment have yet to be established. Therefore, the establishment of evidence-based guidelines for this condition is urgently required.

\section{CONFLICT OF INTEREST}

No potential conflict of interest relevant to this article was reported.

\section{AUTHOR CONTRIBUTIONS}

H.K. participated in data collection, performed the statistical analysis and wrote the manuscript. M.K.L. participated in the study design and data collection. T.H.K. participated in the study design and coordination and helped to draft the manuscript. All authors read and approved the final manuscript.

\section{ORCID}

Hyunji Kim, https://orcid.org/0000-0001-8121-1560

Mi-Kyung Lee, https://orcid.org/0000-0003-1824-476X

Tae-Hyoung Kim, https://orcid.org/0000-0002-0257-3449

\section{REFERENCES}

1. Alfouzan WA, Dhar R. Candiduria: evidence-based approach to management, are we there yet? J Mycol Med 2017;27:293-302.

2. Kobayashi CC, de Fernandes OF, Miranda KC, de Sousa ED, Silva Mdo R. Candiduria in hospital patients: a study prospective. Mycopathologia 2004;158:49-52.

3. Simpson C, Blitz S, Shafran SD. The effect of current management on morbidity and mortality in hospitalised adults with funguria. J Infect 2004;49:248-52.

4. Sobel JD, Fisher JF, Kauffman CA, Newman CA. Candida urinary tract infections--epidemiology. Clin Infect Dis 2011;52 Suppl 6:S433-6.

5. Jain M, Dogra V, Mishra B, Thakur A, Loomba PS, Bhargava A. Candiduria in catheterized intensive care unit patients: emerging microbiological trends. Indian J Pathol Microbiol 2011;54: 552-5.

6. Kauffman CA, Vazquez JA, Sobel JD, Gallis HA, McKinsey DS, Karchmer AW, et al. Prospective multicenter surveillance study of funguria in hospitalized patients. The National Institute for Allergy and Infectious Diseases (NIAID) Mycoses Study Group. Clin Infect Dis 2000;30:14-8.

7. Paul N, Mathai E, Abraham OC, Michael JS, Mathai D. Factors associated with candiduria and related mortality. J Infect 2007; 55:450-5.

8. Magill SS, Swoboda SM, Johnson EA, Merz WG, Pelz RK, Lipsett $\mathrm{PA}$, et al. The association between anatomic site of Candida colonization, invasive candidiasis, and mortality in critically ill surgical patients. Diagn Microbiol Infect Dis 2006;55:293-301.

9. Bougnoux ME, Kac G, Aegerter P, d'Enfert C, Fagon JY; CandiRea Study Group. Candidemia and candiduria in critically ill patients admitted to intensive care units in France: incidence, molecular diversity, management and outcome. Intensive Care Med 2008;34:292-9.

10. Alfouzan WAM. Epidemiological study on species identification and susceptibility profile of Candida in urine. Fungal Genom Biol 2015;5:124.

11. Ghiasian SA, Aghamirian MR, Eshghi GR. Nosocomial candiduria in critically ill patients admitted to intensive care units in Qazvin, Iran. Avicenna J Clin Microb Infec 2014;1:e21622.

12. Brogden KA, Guthmiller JM, Taylor CE. Human polymicrobial infections. Lancet 2005;365:253-5.

13. Garcia-Agudo L, Rodriguez-Iglesias M, Carranza-Gonzalez R. Nosocomial candiduria in the elderly: microbiological diagnosis. Mycopathologia 2018;183:591-6.

14. Bouza E, San Juan R, Munoz P, Voss A, Kluytmans J; Cooperative Group of the European Study Group on Nosocomial Infections. A European perspective on nosocomial urinary tract infections I. Report on the microbiology workload, etiology and antimicrobial susceptibility (ESGNI-003 study). Clin Microbiol Infect 2001;7:523-31. 
15. Lundstrom T, Sobel J. Nosocomial candiduria: a review. Clin Infect Dis 2001;32:1602-7.

16. Lehner T. Systemic candidiasis and renal involvement. Lancet 1964;2:1414-6.

17. Yismaw G, Asrat D, Woldeamanuel Y, Unakal C. Prevalence of candiduria in diabetic patients attending Gondar University Hospital, Gondar, Ethiopia. Iran J Kidney Dis 2013;7:102-7.

18. Garcia-Agudo L, Rodriguez-Iglesias M, Carranza-Gonzalez R. Approach of clinicians to candiduria and related outcome in the elderly. J Mycol Med 2018;28:428-32.

19. Kim J, Kim DS, Lee YS, Choi NG. Fungal urinary tract infection in burn patients with long-term foley catheterization. Korean J Urol 2011;52:626-31.

20. Ozhak-Baysan B, Ogunc D, Colak D, Ongut G, Donmez L, Vural
T, et al. Distribution and antifungal susceptibility of Candida species causing nosocomial candiduria. Med Mycol 2012;50: 529-32.

21. Leendertse $M$, Heikens $E$, Wijnands $L M$, van Luit-Asbroek $M$, Teske GJ, Roelofs JJ, et al. Enterococcal surface protein transiently aggravates Enterococcus faecium-induced urinary tract infection in mice. J Infect Dis 2009;200:1162-5.

22. Cruz MR, Graham CE, Gagliano BC, Lorenz MC, Garsin DA. Enterococcus faecalis inhibits hyphal morphogenesis and virulence of Candida albicans. Infect Immun 2013;81:189-200.

23. Jung SI, Shin JH, Song JH, Peck KR, Lee K, Kim MN, et al. Multicenter surveillance of species distribution and antifungal susceptibilities of Candida bloodstream isolates in South Korea. Med Mycol 2010;48:669-74. 